\title{
Atomistic Visualization of Mesoscopic Whole-Cell Simulations Using Ray-Casted Instancing
}

Martin Falk, Michael Krone and Thomas Ertl

The self-archived postprint version of this journal article is available at Linköping University Institutional Repository (DiVA):

http:/ / urn.kb.se/ resolve?urn=urn:nbn:se:liu:diva-143707

N.B.: When citing this work, cite the original publication.

Falk, M., Krone, M., Ertl, T., (2013), Atomistic Visualization of Mesoscopic Whole-Cell Simulations Using Ray-Casted Instancing, Computer graphics forum (Print), 32(8), 195-206.

https:// doi.org/ 10.1111/ ogf.12197

Original publication available at:

https:/ / doi.org/ 10.1111/ cgf.12197

Copyright: Wiley (12 months)

http:/ / eu.wiley.com/WileyCDA/ 


\title{
Atomistic Visualization of Mesoscopic Whole-Cell Simulations using Ray-Casted Instancing
}

\author{
Martin Falk, Michael Krone, and Thomas Ertl
}

Visualization Research Center (VISUS), University of Stuttgart, Germany

$\{$ martin.falk|michael.krone|thomas.ertl\}@ vis.uni-stuttgart.de
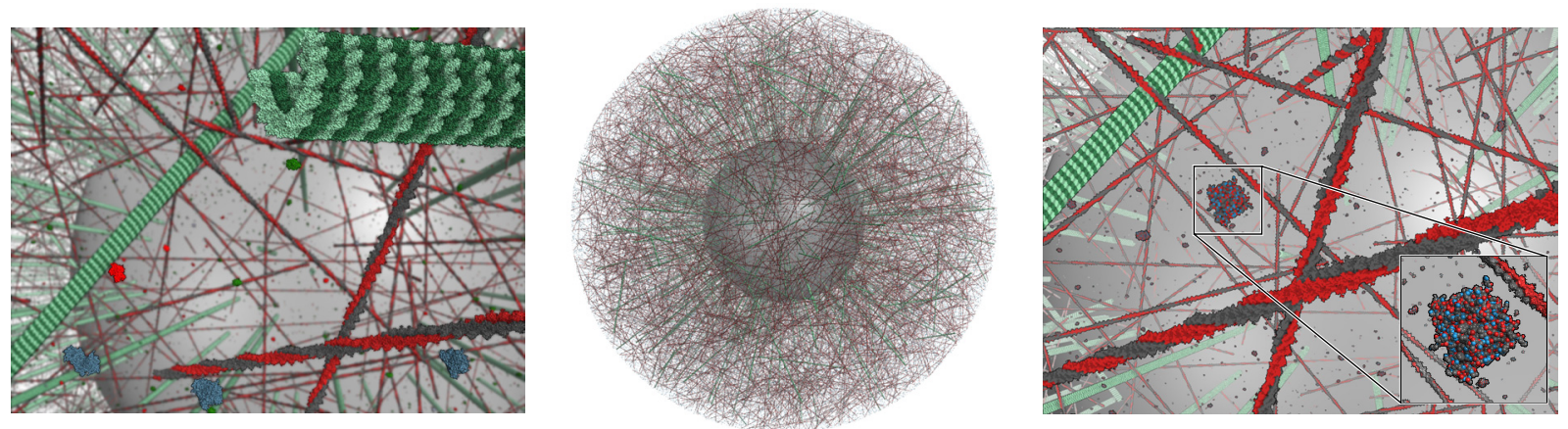

Figure 1: Simulation of the ERK pathway within a simplified cell model (25 billion atoms, center). With our optimized visualization algorithm, it is possible to render this data set at $3.6 \mathrm{fps}$. Left: close-up view rendered with depth of field. Right: close-up view showing the cytoskeleton and signal proteins. For molecules near the camera, individual atoms are discernible.

\begin{abstract}
Molecular visualization is an important tool for analyzing the results of biochemical simulations. With modern GPU ray casting approaches it is only possible to render several million of atoms interactively unless advanced acceleration methods are employed. Whole-cell simulations consist of at least several billion atoms even for simplified cell models. However, many instances of only a few different proteins occur in the intracellular environment, which can be exploited to fit the data into the graphics memory. For each protein species, one model is stored and rendered once per instance. The proposed method exploits recent algorithmic advances for particle rendering and the repetitive nature of intracellular proteins to visualize dynamic results from mesoscopic simulations of cellular transport processes. We present two out-of-core optimizations for the interactive visualization of data sets composed of billions of atoms as well as details on the data preparation and the employed rendering techniques. Furthermore, we apply advanced shading methods to improve the image quality including methods to enhance depth and shape perception besides non-photorealistic rendering methods. We also show that the method can be used to render scenes that are composed of triangulated instances, not only implicit surfaces.
\end{abstract}

Categories and Subject Descriptors (according to ACM CCS): I.3.7 [Computer Graphics]: Three-Dimensional Graphics and Realism-Raytracing, I.3.6 [Computer Graphics]: Methodology and Techniques-Graphics data structures and data types, J.3 [Computer Applications]: Life and Medical Sciences-Biology and genetics

\section{Introduction}

The interactive rendering of large particle systems has been an active area of research for many years. One of the most prominent areas of application is the visualization of the re- sults from particle-based simulations. The data sets coming from this domain have been rapidly increasing in size over the last years. Simulation data sets already push current graphics hardware to its limits. As the data sets grow, the vi- 
sualization must keep pace to enable researchers to analyze the simulations. One possible and popular approach is to use compute clusters for visualization. From our perspective, it is important to enable the visualization of large particle data sets on common desktop workstations. Our main focus lies on the visualization of biomolecular data sets. More precisely, we want to visualize the results from coarse-grained (so-called mesoscopic) particle-based simulations of whole cells. Even though the simulations operate on single particles representing whole molecules, our goal was to visualize the simulation on an atomic scale (cf. Figure 1). This can help scientists to see the detailed function of cellular processes and gain a better understanding of cellular dimensions and complexity. While atom-based simulations typically range up to only tens of millions of atoms, visualizations of mesoscopic simulations can easily reach tens of billions of atoms if shown on an atomistic level.

This paper is an extended version of our work presented at the last workshop for Visual Computing for Biology and Medicine (VCBM) [FKE12]. The main contribution of our work is a method for interactive visualization of mesoscopic whole-cell simulations on an atomistic level of detail. Our work builds on the recent work of Lindow et al. [LBH12]. They visualize molecular structures reconstructed from electron tomography images. Here, many instances of the only few individual molecules occur. Lindow et al. proposed a fast ray casting method to render these instances. The molecules are rendered using the Van-derWaals $(\mathrm{VdW})$ model where each atom is represented by a sphere.

As in the work of Lindow et al. [LBH12], our data sets consist of large numbers of instances of only few individual molecules. We use an extended and optimized version of their algorithm to visualize our dynamic data sets resulting from simulations. The first optimization is a hierarchical rendering approach that also leads to an implicit Level of Detail (LoD). The second optimization is a depth culling method that does not rely on sorting. Furthermore, we wanted to draw arbitrary molecular models besides the VdW model. Thus, we expanded the method to render triangulated objects. This way, we can use advanced molecular models like molecular surfaces. Our visualization shows the underlying data of the simulation and provides new, detailed insights into the simulated system. In addition, it can be used for artistic reasons and educational purposes, showing the crowded internal environment of the cell. Therefore, we show the applicability of various image enhancement techniques to create renderings that are visually pleasing and, moreover, highlight the structure and spatial relationships in the data. Our visualization is interactive for giga-scale particle data sets on consumer graphics hardware. Furthermore, we show the applicability of the method to triangulated objects and the feasibility of illustrative methods to enhance the data analysis and image quality.

\section{Previous Work}

When dealing with huge particle data sets, parallel visualization approaches are a reasonable and, in most cases, promising approach. Popular visualization frameworks like ParaView [LHA01] support parallel visualization. As stated in the introduction, our work focuses on visualizations using commodity desktop workstations equipped with highend consumer graphics hardware. In the following, we therefore focus on the previous work from this area.

Simple glyph-based visualization of particle data sets is a flexible and powerful representation for data analysis. Most commonly, particles are rendered as spheres or ellipsoids, where features can be mapped to visual qualities like the radius or color of a sphere. Gumhold [Gum03] introduced the concept of point-based GPU ray casting to render large numbers of ellipsoids interactively. This method can be seen as the de-facto standard for rendering quadrics nowadays since it is vastly superior to classical triangle-based geometry in terms of speed and image quality. Recently, Grottel et al. [GRE09] showed that it is possible to render millions of spheres interactively on modern GPUs without advanced acceleration techniques. They also evaluated different ways to upload the data to the GPU for rendering, which today is one of the major bottlenecks for visualizing large, dynamic data sets. Subsequently, they extended their method using a twolevel occlusion culling and deferred shading [GRDE10]. With this optimized technique, they have been able to render data sets of up to 100 million particles interactively. However, as with all occlusion culling methods, their algorithm is only beneficial for dense data sets with a large amount of occluded geometry. This is not the case for our data sets since the interior of our simulated cell is rather sparsely populated due to the simplified simulation model.

Recently, Lindow et al. [LBH12] presented a method to render biomolecular data sets comprising over a billion atoms. Since they visualize molecular structures reconstructed from electron tomography (ET) images, their data sets consist of many instances of the same molecules. Due to the limited resolution of ET images it is only possible to identify individual molecules or molecular complexes, but not to extract the spatial structure or even individual atoms. Therefore, the same model is used to visualize all molecules of a certain species. Their visualization method exploits this fact to achieve interactive rendering performance. The general idea of their algorithm can be outlined as follows: Each molecular model is uploaded to the GPU memory during the preprocessing stage. During rendering, instances of the bounding boxes of the uploaded models are rendered for each molecule. For each fragment of the bounding boxes, a ray is cast into the scene and intersections with the individual atom spheres are computed for rendering. Here, a grid-based acceleration structure is traversed, analogical to classical GPU volume ray marching [KW03, HSS*05]. In Section 5, we give a detailed description of the original al- 
gorithm and explain our extensions and optimizations. A similar approach was presented by Lampe et al. [LVRH07] to visualize large proteins. They assemble the proteins on the GPU after transferring only the position and rotation of each amino acid. The individual atoms per amino acid are stored in a texture. The points for the atoms are generated and transformed in the geometry shader and rendered using ray casting in the fragment shader. In contrast to [LBH12], this method is limited by the number of primitives that can be emitted by the geometry shader.

Triangular objects are typically rendered using the rendering pipeline of the GPU. Even though the GPU pipeline is programmable nowadays, it is still optimized for triangle rendering. Furthermore, modern GPUs support instancing to render multiple copies of an object efficiently. However, Grottel et al. [GRZ* 10] showed that GPU ray casting can be beneficial for large numbers of polygonal objects. They rendered large numbers of crystallites and obtained higher frame rates compared to triangulated instances. In this case, though, they only had to compute ray-plane intersections. For a more general case, actual ray-triangle intersections have to be computed. Möller and Trumbore [MT97] presented a highly efficient ray-triangle intersection test. The comparison by Shumskiy and Parshin [SP12] showed that it is also most efficient for GPU ray tracing. Rendering large numbers of geometric instances has also been achieved in real-time using cluster-based CPU ray tracing [Wa104]. In contrast, our technique is designed to run on a single desktop workstation, as mentioned above. However, the GPU ray casting [LBH12] is technically related to ray tracing. While real-time ray tracing needs extensive acceleration structures, our method exploits the graphics pipeline.

Instead of using ray-triangle intersections for displaced or feature-rich geometry in large scenes, Laine and Karras [LK10] propose a voxel-based approach obtaining a performance comparable to triangle ray casting. The entire scene, including necessary information for illumination and shading, is stored in a sparse voxel octree. To avoid artifacts caused by voxelization surface approximations are used. However, the voxel octree has to be generated in an expensive pre-processing step. Another approach for rendering large numbers of triangles is point-based rendering. Wand et al. [WFP*01] presented the randomized z-buffer method which samples relatively low numbers of surface points and renders them interactively. The fragments in between the points are interpolated. This can lead to visible artifacts in the final image. Apart from that, the method makes use of caching for fast rendering. If the scene changes, a costly resampling of the points has to be performed.

\section{Biological Background}

The multidisciplinary field of systems biology investigates the characteristics and the complex interactions of all elements in a particular biological system [Pal00]. Methods from systems theory are used to build mathematical models of processes within an organism. These models are tested either with in-silico simulations or heuristics. Afterwards, the model results are then compared with the data obtained in experiments. In the context of our work, signaling processes in the cellular environment are of special interest.

We approximate the complex interior of a biological cell for in-silico simulations as described by Falk et al. [FKRE09]. The plasma membrane defines the outer boundaries of the cellular environment and has a spherical shape. The nucleus, which contains the DNA, is located in the interior of the cell. The remaining space inside the plasma membrane, the cytoplasm, is filled with various compartments, proteins, hormones, and water molecules, which are the most abundant species. The number of proteins of the same species in a single cell ranges from only a few to billions. For example, there are about 500 billion actin molecules in a liver cell. The structure of the cell is maintained by the cytoskeleton, a scaffold built of three different filament types. Microtubules exhibit a cylindrical form of thirteen protein columns. Their main functions are the stabilization and movement of the cell and the intracellular transport of proteins. Actin filaments, also known as microfilaments, stabilize the cell and withstand both tensile and compressive forces. The intermediate filaments, the third filament type, build a tightly connected network around the nucleus. They serve as fixture for organelles in the cytoplasm. All three types of filaments are represented by elongated cylinders. Proteins in the cytoplasm move by diffusion and through the transport along microtubules and actin filaments with the aid of motor proteins.

Molecular dynamics simulations are not suitable to simulate such a cellular environment on the atomic scale because they only cover short time scales (picoseconds to milliseconds [SMLL*10]) and smaller systems. Such timescales are too small to reproduce the effects of cellular signaling mechanisms, which take place in seconds to even hours. To address this problem, mesoscopic simulations can be employed. When neglecting atomistic effects, the atomic structure of a signaling molecule can be replaced by a sphere with the hydrodynamic radius of the physical molecule. We use a three-dimensional agent-based stochastic simulation employing CUDA for GPU acceleration [FOE*11] where each agent represents one single molecule. This type of simulation also considers spatial effects like asymmetric protein distributions in contrast to models solely based on differential equations, which use average concentrations [PSQH06]. The model includes chemical interactions between molecules, diffusion, and structural interactions like motorized transport along cytoskeletal filaments.

\section{Data}

The atomic structures of all molecules used in this paper are obtained from the protein data bank (PDB) [BWF*00]. The 

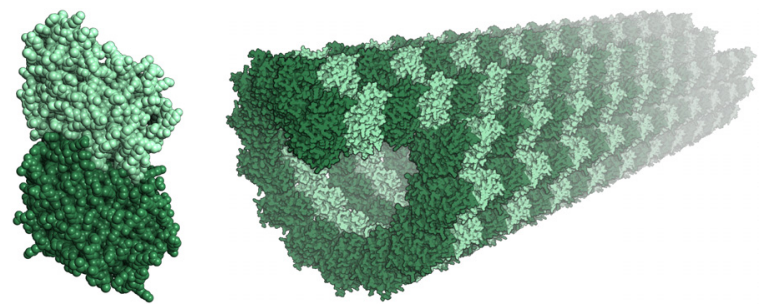

Figure 2: Structure of microtubules. Left: $\alpha$-tubulin (dark green) and $\beta$-tubulin (light green). Right: thirteen complexes of $\alpha$ - and $\beta$-tubulin are arranged in a circle building one turn of a microtubule.

data files provide information on the location and type of atoms as well as conformal information.

Proteins can usually be loaded from a single file. However, in some cases a file might contain only a part of the protein which then has to be combined with other protein parts.

Microtubules consist of $\alpha$ - and $\beta$-tubulin, which form dimers (Figure 2). Thirteen dimers (PDB-ID: 1TUB) arranged in a short helical ring represent one row of the microtubule. The dimer itself is about $8 \mathrm{~nm}$ high and is continuously shifted along the microtubule axis reaching $12 \mathrm{~nm}$ per turn $\left[\mathrm{LBK}^{*} 07\right]$. The resulting seam on one side of the tubule can be seen in Figure 2, right.

Actin filaments consist of actin monomers (PDB-ID: 3MFP) binding at the tip of a growing filament. Figure 4 shows one complete turn of the helical structure built by 28 actin molecules. The length of one turn is $77.3 \mathrm{~nm}$. The helix can be constructed either with the transformation provided in the PDB entry or the model of Holmes et al. [HPGK90].

\section{Algorithm}

The rendering method we are using relies on the fact that, even though the overall number of particles in the scene may be as high as several billions of atoms, the number of individual molecules is typically quite low. That is, the atom positions of each type of molecule have to be stored only once and can be rendered for all instances of the same molecules. This reduces the number of atoms which have to be stored in main or graphics memory to at most a few millions. In the following, we explain the rendering technique presented by Lindow et al. [LBH12] onto which our work is based on. We also go into detail about our extensions and optimizations to the original work.

Molecule Setup and Instancing. As outlined in Section 3, our primary sources of data are mesoscopic simulations which do not operate on individual atoms but on whole molecules. Therefore, all molecules in the scene are rigid, that is, they are only translated and rotated, but do not undergo any internal deformations. Consequently, we have to transfer the atomistic molecular models only once per protein type to the GPU memory-just like the original algorithm. During rendering, we can draw multiple instances of the same molecule. This allows keeping the amount of data which has to be transferred for each render pass from the CPU to the GPU very low. For each molecule we render, we only have to transfer the translation and rotation which can either be stored in a single matrix or in a quaternion and an additional translation vector.

For the microtubules we use two instance blocks, one with ten rows consisting of 130 tubulin dimers resulting in $882 \mathrm{k}$ atoms and one with twenty rows $(1.76 \mathrm{M}$ atoms). The two blocks of the actin filaments consist of 28 actin molecules ( $82 \mathrm{k}$ atoms) and 56 molecules ( $164 \mathrm{k}$ atoms) respectively. The blocks of actin filaments or microtubules are fitted on the filaments of the cytoskeleton from the simulation.

Grid Traversal and Ray Casting. To allow for fast, highquality rendering of the atoms, we employ ray casting to compute the intersections between the viewing rays and the atoms. A uniform grid is used for space subdivision to reduce the number of intersection tests. All atoms of a molecule are sorted into this grid (Figure 3, left). A boxsphere intersection is used to determine all cells of the grid with which an atom intersects. To store the data of the uniform grid we use the grid data structure proposed by Lagae and Dutré [LD08].

Each cell of the grid is mapped onto one voxel of a 3D texture. The atomic data-i.e. position, radius, and color IDsare stored in two additional 2D textures (Figure 3, right). The first texture contains the atom position and its radius represented in 32-bit floating point values. The color identifiers, e.g. atom type, chain ID, or strand ID, are stored in the second texture with up to four channels. This additional data is accessed via a $2 \mathrm{D}$ index stored in the $3 \mathrm{D}$ grid texture. In

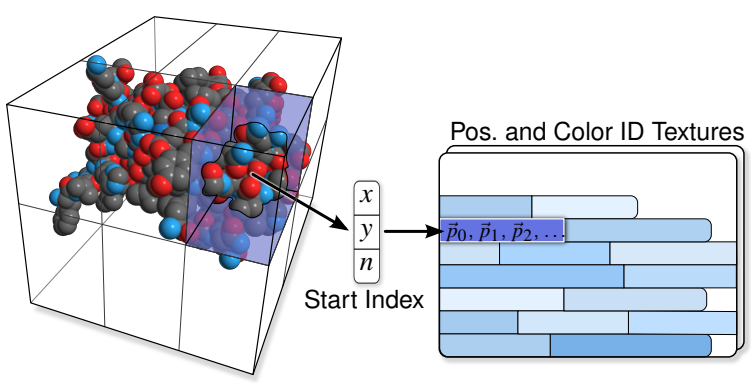

Figure 3: The protein is embedded into a uniform $3 D$ grid, where each grid cell refers with an index to a consecutive list of atoms intersecting with this cell. The lists containing the atom information, i.e. atom position, radius, and coloring, are stored in two $2 D$ textures. 


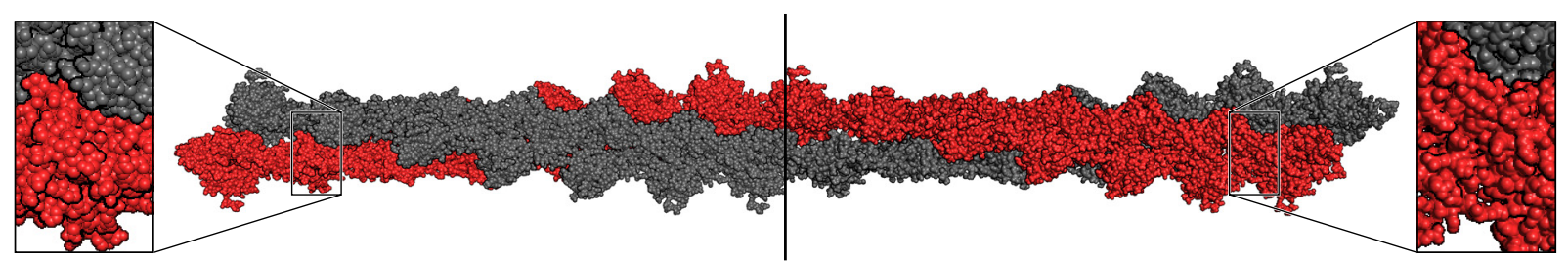

Figure 4: One turn of an actin filaments consists of 14 layers of 2 actin monomers each. For clarity we color the two differently in order to highlight the pitch. The normal approximation (right) enhances the global structure compared to the shading with exact normals (left).

addition to the $2 \mathrm{D}$ index, each voxel also contains the number of atoms in this cell. Upon rendering the atoms, only the grid's bounding box of each instance is drawn. By rendering only the back faces of the bounding box and computing the corresponding position on the front side, the ambiguity between front and back faces is avoided. The fragment shader computes the view ray through each fragment covered by the bounding box and traverses the grid cells front to back. The individual ray-sphere intersections for the atoms are consequently computed per grid cell. As soon as at least one atom is hit, the grid traversal can be stopped. Note that it is important to compute all intersections in one grid cell to obtain the closest intersection since the atoms are not ordered.

Lindow et al. [LBH12] observed that a ray-voxel traversal [AW87] is superior to a ray-layer traversal, where several grid cells have to be considered for each step along the view ray. One drawback of the former method is that the atoms have to be replicated for each grid cell they intersect. Another drawback is that a change of atom radii requires a recomputation of the grid, which is not necessary when using ray-layer traversal. Since this is not needed in our application, we decided to use the faster ray-voxel traversal. If the size of the grid cells is chosen with respect to the atom size, one can ensure that a single atom is contained in at most eight grid cells. Moreover, the better rendering speed outweighs the additional memory consumption, which is not a problem on current GPUs with $1 \mathrm{GiB}$ or more of graphics memory. Lindow et al. proposed a grid where the number of voxels equals the number of atoms. We chose the size of the grid cells with respect to the atom size, ensuring that a single atom is contained by at most eight grid cells. Hence, a voxel size of 4 Ångström was chosen. In our experience, this also gives the best performance. We observed that in this case the resulting number of voxels roughly corresponds with the number of atoms, that is, we concur with Lindow et al.

Deferred Shading. Similar to Grottel et al. [GRDE10], Lindow et al. [LBH12] use deferred shading. This not only results in a slight speedup, but also enables the normal correction scheme used in both works to smooth out high frequencies between adjacent normals of distant objects. This results in a more continuous lighting which creates a surfacelike impression.
Grottel et al. [GRDE10] propose to use deferred shading with different normal calculations depending on the distance from the camera. For near objects, the analytically computed normals are used for shading. As the objects move away from the camera an approximated normal is used. The approximated normal is the normal of the center point of a quadratic Beziér surface over the current point and its neighbors. A smooth transition between analytical and approximate normal is obtained by linear interpolation. Lindow et al. [LBH12] extended this approach using a radius function for the approximated normal to influence the smoothness. They also use the inverse view direction as normal for distant fragments. Figures $4 \& 5$ show a comparison between analytical and approximated normals.

\subsection{Optimizations}

Depth Culling. In classical polygon-based computer graphics, the OpenGL pipeline can reduce the computational load by the early $\mathrm{z}$ test, which takes place after the vertex processing stage: If a fragment which is closer to the camera than the current fragment was already rendered, the computation for the current fragment can be aborted. However, the early $\mathrm{z}$ test is automatically disabled if a fragment shader manipulates the depth of the fragment or uses discard to reject fragments. We actually need both functions: We need the correct per-fragment depth values for all spheres for molecule-molecule intersections and we must discard fragments where a ray traverses the bounding box of an object but does not write any output because no atom is hit.

We propose to store the depth of the closest intersection and reuse it in the fragment shader to perform an early reject. If the stored depth value is smaller than the depth of the bounding box front, the computation in the fragment shader can safely be discarded. In case the front position is closer the grid traversal takes place and, if an intersection is found, the resulting depth is stored into a second texture. The two textures with the depth values are swapped in a ping-pong fashion after $n$ molecule instances have been drawn. The regular depth buffer is still necessary to ensure the correct depth ordering of the $n$ instances of one pass. Since we assume the molecules to be randomly distributed in our cell $n$ can be large and the optimization is still beneficial (see Section 6). 

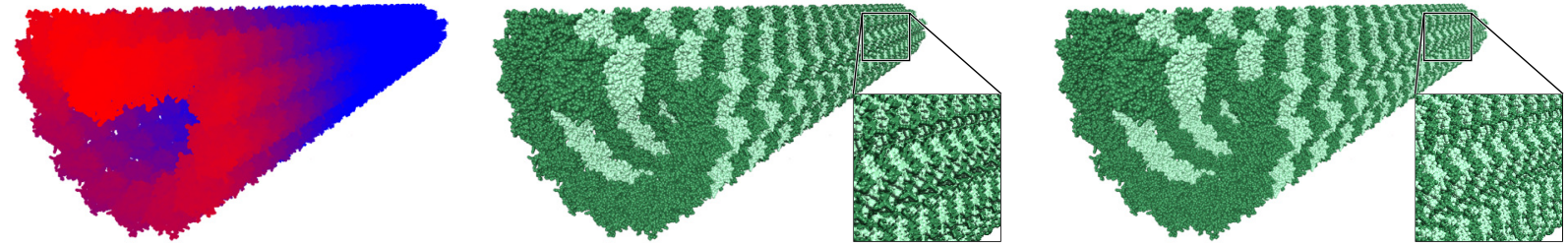

Figure 5: Normal estimation along a microtubule. Left: interpolation between analytical normal (red) and averaged normal (blue) depending on the camera distance. Center: the roughness of the surface is revealed with normal estimation. Right: without normal estimation. Note, only direct illumination is applied.

To avoid a second render target, which would slow down the rendering, we combine the depth information with the normal and the color ID resulting from the ray-sphere intersections. By using integer color IDs, we can save the scaled third component of the normal together with the color id and are able to reconstruct the normal in the deferred shading. Therefore, it is possible to store the normal, the color ID, and the closest depth in a single texture. In the subsequent deferred rendering, both result textures are sampled and the texture with the smaller depth value is chosen.

Hierarchical Ray Casting When visualizing our simulation results, most of the molecules will only cover a few pixels on the final image. The resulting artifacts can be remedied by the approximate normal approach described above. But this does not reduce the computation workload for rendering. Hence, we extend the original algorithm of Lindow et al. [LBH12] by a hierarchical ray casting. If, during the grid traversal, a grid cell covers only a single pixel, we can omit the ray-sphere intersection tests, because it is impossible to distinguish individual spheres anyway. In this case, our algorithm only makes one texture lookup to determine whether the grid cell is empty or not. If the grid cell is not empty, this will be considered as a hit and the grid traversal will be stopped. This is only valid because the grid cells have roughly the same size as an atom. Therefore, the probability of an atom being hit by a ray traversing the corresponding grid cell is high. The result is basically a binary voxelization of the data set. The same approach can be used for whole molecules if the bounding box of the molecule covers only one pixel on the image plane. Figure 6 illustrates the principal approach for a single viewing ray. The different levels of detail when hierarchical ray casting is applied are highlighted in Figure 7. Since the normals of far-away atoms are approximated during deferred shading, the binary voxelization of the grid does not show up in the final image. This technique is especially feasible for our simulation data sets where the cytoskeleton is composed of very large molecules and the signal proteins are quite small in comparison. Note that this has no effect on the deferred shading, since we only use approximated normals for distant objects. The approach also prevents molecules from being

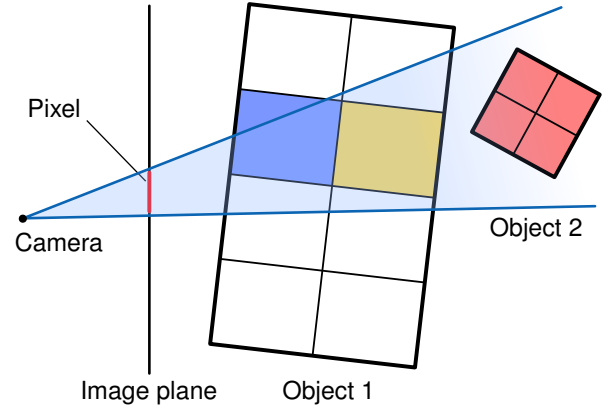

Figure 6: With the hierarchical ray casting approach intersection tests are omitted for grid cells covering only a part of a single pixel (yellow) in contrast to partially covered voxels (blue). No grid traversal is necessary for object 2 since its bounding box (red) fits entirely into the pixel.

invisible because they would be smaller than one pixel and not be hit by the view rays.

The size of the pixel is calculated for the depth at the end of the current view ray. With the aid of the intercept theorem the pixel size is obtained from the field of view, the viewport height in pixels, and the corresponding depth. Since no intersection tests are performed when the pixel is larger than a grid cell, a special coloring scheme has to be employed. We propose to use the color ID of the first entry of the cell. This has the advantage, that coloring according to chain, strand, or instance ID is still possible and also visible. Other possible color schemes could comprise of a precomputed averaged color per grid cells or just a single color predefined per molecule instance.

\subsection{Extension to Triangular Meshes}

Current GPUs are capable of rendering a few million triangles at highly interactive frame rates. This is in particular the case when the mesh data resides in GPU memory. For repetitive data like protein surface meshes placed at the protein locations, instancing can be used. However, when considering the total number of proteins, the triangle count exceeds 


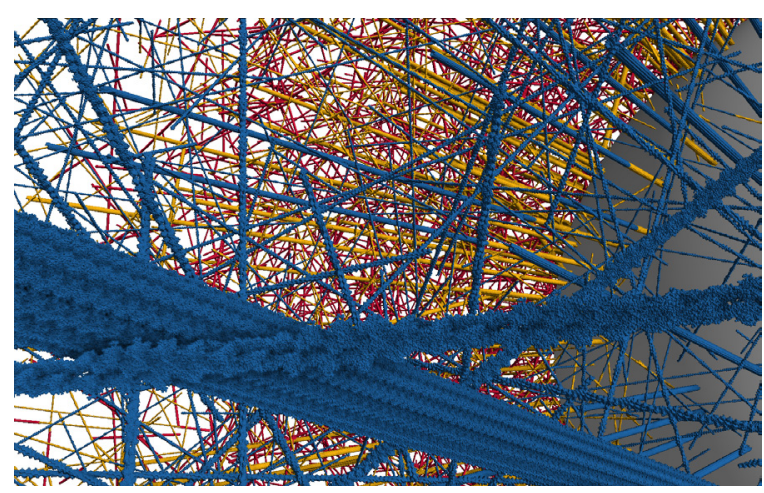

Figure 7: Hierarchical ray casting provides full detail (blue) in the foreground, filled grid cells (yellow) in the medium distance, and filled molecule bounding boxes covering only parts of a pixel (red) in the back. Pixel sizes are exaggerated so that the effect becomes visible.

several million triangles even with conservatively-sized, low resolution meshes. To improve the performance, the idea of grid-based ray casting is applied to triangle meshes. As for the atomic structures, this method relies on a large amount of identical meshes.

The algorithm has to be modified only slightly in order to render objects represented by triangle meshes instead of implicitly described, spherical atoms. Instead of storing spheres in the $3 \mathrm{D}$ grid, the individual triangles of the mesh are inserted. A fast triangle-box intersection test [AM01] is used to determine with which grid cells each triangle intersects. The triangle data, i.e. the vertices, their vertex normals, texture coordinates, and colors are stored in three $2 \mathrm{D}$ textures. Storing and duplicating the three vertices of each triangle explicitly increases the memory footprint but avoids costly texture indirection by means of indices.

Since the triangles of the mesh deviate much more in size than the sphere radii of the atoms, the resolution of the $3 \mathrm{D}$ grid has to be chosen carefully. For optimal performance, the grid is adjusted according to the tesselation of the mesh. If the grid is too coarse, the less grid cells have to be traversed but the average workload per voxel is increased as more triangles have to be tested for intersections. On the other hand, in a fine-granular grid the voxels hold only few triangles each and the traversal costs rise. We therefore choose an individual grid resolution per molecule so that 20 to 30 triangles are stored in a voxel at most which is in our experience a good trade-off.

The grid traversal during ray casting remains unchanged except for the intersection test which is replaced by an efficient ray-triangle intersection test [MT97]. The barycentric coordinates resulting from the intersection test are used to interpolate the normals and the color values. If texturing is enabled, color textures can be sampled with the interpolated texture coordinates. Since integer color IDs cannot be interpolated reasonably, the color value has to be stored in a separate render target in addition to the normal and the current depth. The alpha channel of a texture is optionally used for masking transparent parts that can be used for see-through effects. Please note that the technique supports only binary opacity, i.e. either fully opaque or completely transparent materials. Semi-transparency could be used for single objects. However, during ray traversal the intersections in each grid cell have to be sorted according to depth to yield correct blending results. This would be computationally costly and lead to a loss in performance. Furthermore, if two or more object instances intersect each other depth sorting is not possible because each instance is ray marched independently.

We extended our application so that it can load meshes in the Wavefront OBJ file format. This file format is widely used in many modeling and animation packages. It consists of the OBJ file which stores the vertices, normals and primitives (e.g. triangles or quadrilaterals) and an optional MTL file which stores material information like colors and textures. We used the free molecular visualization program VMD [HDS96] to generate molecular surface meshes. VMD supports the OBJ file format for storing meshes.

\subsection{Shading and Postprocessing}

We use deferred shading [ST90] for the final image generation. One of the most important features of deferred shading is that it limits lighting computations only to visible fragments. This is not only advantageous because it reduces the computational load, it also allows us to use various post-processing techniques in image-space. Using the atomistic representation, the user can apply the common coloring schemes for molecular graphics. For the proteins, we implemented coloring by element, by molecular subunit or chain, and by instance ID. For the cytoskeleton, the user can additionally select coloring per strand. Triangle meshes are colored either according to vertex colors or texture values, respectively.

We allow the user to choose between a variety of illustrative methods to enhance the final image. All methods have been chosen to facilitate the visual analysis of the renderings as well as for artistic reasons. Artistic renderings, which resemble a hand-drawn illustration, are often easier on the human perception. A prominent example for this are the Molecule of the Month renderings by the renowned illustrator David Goodsell [Goo] and the work on enhancing real-time molecular graphics by Tarini et al. [TCM06]. Likewise, we added the option to apply depth-dependent silhouettes [ST90]. Silhouettes are a simple, yet effective cue to emphasize spatial differences and to separate objects.

Depth of field is an effect from photography which separates foreground and background since only objects in the focal plane are sharp whereas everything out of focus is 


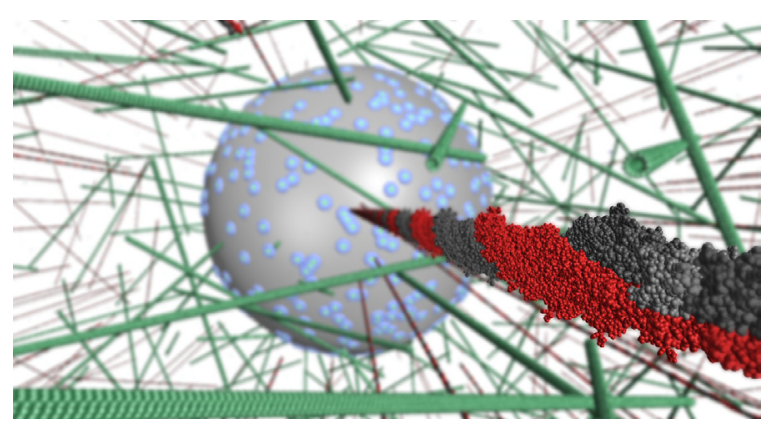

Figure 8: Depth of field applied to a sparse test scene (10 billion atoms). The red actin filament (right) is in focus.

blurred (cf. Figures $1 \&$ 8). In visualization, this can be used to draw the attention of the user to a specific region. The field in focus gets shallower as the focused object gets closer to the camera. Consequently, far away focal points lead to a broad field in focus. To obtain the depth of field effect, we create a mipmap chain of the color and depth buffer of the scene. The circle of confusion $(\mathrm{CoC})$ is then used to sample the mipmap chain. The size of the $\mathrm{CoC}$, which is a circle in the image plane, depends on the depth of the fragment, the aperture, and the focal length (refer to [PC81] for details).

The commonly used local lighting can be confusing and lead to artifacts when rendering a huge number of small spheres. Toon shading is a non-photorealistic rendering technique that shows discrete steps in the coloring instead of a smooth gradient. We allow the user to switch between local lighting, toon shading, and flat shading. Additionally, the user can use screen space ambient occlusion (SSAO) [Kaj09] to enhance depth perception. Due to the lack of lighting, flat shading offers no shape cues for the rendered spheres. However, in combination with the depthdependent silhouettes and a toon-shaded variant of SSAO, flat shading results in a very clear and useful visual appearance (cf. Figure 9).

\section{Results}

We integrated the atomistic rendering method in our visualization program which is used by our project partners to analyze the results of the mesoscopic simulations. A screenshot of the application is shown in Figure 10. The user can load the simulation data sets into the program and select different views to analyze the results. The main view in Figure 10 shows the atomistic visualization with the cytoskeleton, proteins, and the nucleus. The user can also select graph views like the one bottom right which show for example the radial concentration of a certain kind of protein. The application was designed in collaboration with the users in order to maximize the usability and user-friendliness and the serviceability for the intended visual analysis.
We measured the performance of our visualization using data sets from our mesoscopic simulation and molecular surface meshes. The simulation setup and the resulting data set are described in section 6.1. The results of our measurements are given in section 6.2. Section 6.3 discusses the performance of triangle rendering using molecular surface meshes.

\subsection{ERK Pathway Simulation}

The mitogen-activated extracellular-signal-regulated kinase (ERK) pathway is responsible for cell growth, proliferation, and differentiation. Growth factors initiate the signal cascade from Raf over MEK to ERK. The ERK pathway is modeled in our system as follows. The upstream part of the pathway, B-Raf (PDB-ID: 3PPJ), is located near the plasma membrane and always active. MEK1 (PDB-ID: 1S9J) on the second tier is activated by BRaf via double-phosphorylation, i.e. adding two phosphate groups. The activated form of MEK1 activates the final protein, which is ERK1 (PDB-ID: 1ERK). Activated proteins might be deactivated by phosphatases, here MKP3 (PDB-ID: 1MKP), by removing the phosphate groups.

The diameter of the plasma membrane was set to $10 \mu \mathrm{m}$ and the diameter of the nucleus in the center to $4 \mu \mathrm{m}$. Table 1 lists the components of our simulated cell. About 250,000 proteins are present in the beginning and the total amount keeps almost constant over the course of time. The simulation of $7 \mathrm{~s}$ with a step width of $\Delta t=50 \mu \mathrm{s}$ took about two hours on an NVIDIA GeForce GTX 560. The mesoscopic simulation approximates molecules by spheres. Hence, rotational diffusion is not modeled explicitly, but instead incorporated into the reaction rates. To account for the random rotation of proteins in the visualization, we mimic rotational diffusion by applying random rotations onto each molecule when the particular time step is loaded. For consecutive time steps, the rotational perturbation is incrementally applied to

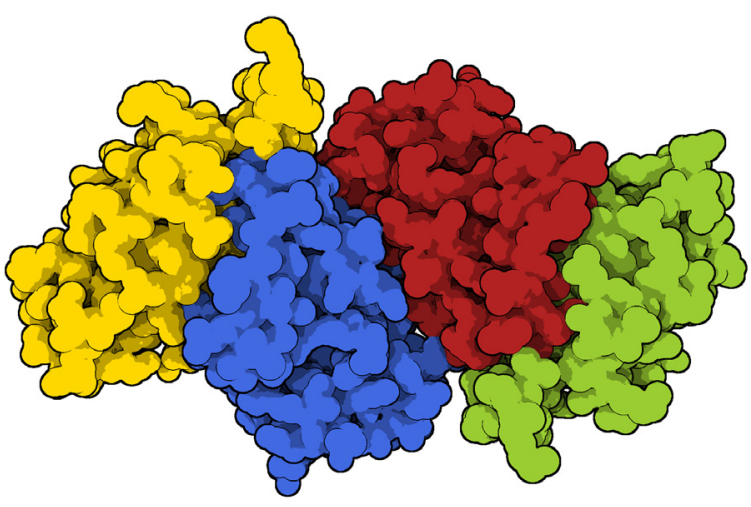

Figure 9: Non-photorealistic rendering of a protein, colored according to the chain, using flat shading, silhouettes, and toonified screen space ambient occlusion (PDB-ID: 3PPJ). 


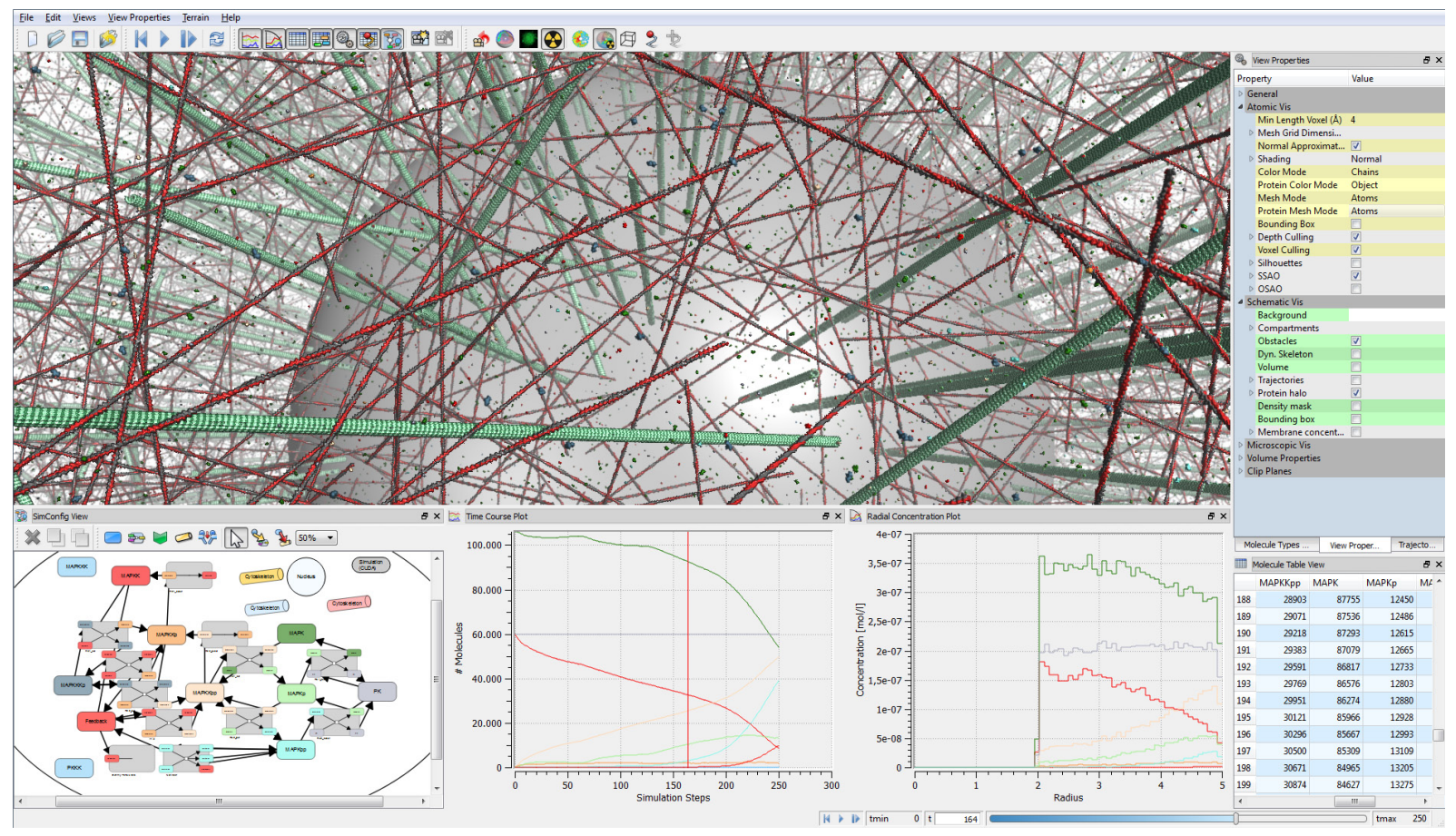

Figure 10: The visualization application used for our work. The framework supports different data views which can be freely arranged. The top view shows the atomistic visualization of the simulation data, the lower left view shows the cellular simulation setup, and the bottom right is a graph view. The parameters are listed in the panel to the right. Users can create additional views to show other data values or visualization styles.

ensure a smoother appearance. The molecule position and rotation between two time steps is computed using linear interpolation. In Figure 1, the results of our visualization applied to the ERK pathway simulation data set are shown.

\subsection{Simulation Rendering Performance}

We measured the performance of our method using various data sets from real molecular dynamics simulations. Our test system was an Intel Core i7 $920(4 \times 2.6 \mathrm{GHz})$ with $6 \mathrm{GiB}$ RAM and a NVIDIA GeForce GTX 580 (1.5 GiB RAM). We used a grid cell size of $4 \AA$. For the depth culling, the number of proteins per iteration was set to 4,096 , which turned out to

Table 1: Parameters of the components of the ERK model. The letter $\mathrm{B}$ denotes billions $\left(10^{9}\right)$.

\begin{tabular}{lrrrrr}
\hline Type & \# Elems. & Length & \multicolumn{2}{c}{ \# Instances } & \# Atoms \\
& & {$[\mu \mathrm{m}]$} & long & short & \\
\hline Actin & 7,500 & 9,873 & 62,051 & 3,622 & $14.34 \mathrm{~B}$ \\
Tubules & 800 & 1,303 & 7,954 & 378 & $10.44 \mathrm{~B}$ \\
ERK & 246,000 & - & - & - & $0.59 \mathrm{~B}$ \\
\hline Total & & \multicolumn{4}{c}{$25,421,804,076=25.42 \mathrm{~B}$} \\
\hline
\end{tabular}

give the best results. The view resolution was $1920 \times 1200$. We adjusted the camera so that most of the screen was covered by the cell and zoomed in so that the proteins in the foreground were not rejected by hierarchical ray casting. As with all ray casting methods, the rendering performance is highly depending on the camera adjustment and the number and size of objects in the scene. Therefore, we will not conduct a formal comparison with the performance values given by Lindow et al. [LBH12] but rather compare our optimizations to the original algorithm.

For the huge data set containing more than 25 billion atoms, we measured $1.8 \mathrm{fps}$ without any optimizations. With our custom depth culling enabled, the frame rate was $2.2 \mathrm{fps}$ which is about $20 \%$ faster. With the hierarchical ray casting and no depth culling, we measured $3.3 \mathrm{fps}$ (about $80 \%$ faster). With hierarchical ray casting and depth culling, the frame rate was at $3.6 \mathrm{fps}$, which is twice as high as the nonoptimized version. Zooming further into the scene similar to Figure 1 (right) led to an increase in the rendering performance to about $8 \mathrm{fps}$. The rendering of the whole cell, as depicted in the center of Figure 1, was possible with $3.6 \mathrm{fps}$ at a height of 1200 pixels when enabling all optimizations. The data set was rendered at $10.3 \mathrm{fps}$ when covering only half the viewport height and $21.8 \mathrm{fps}$ at a third of the height. We 


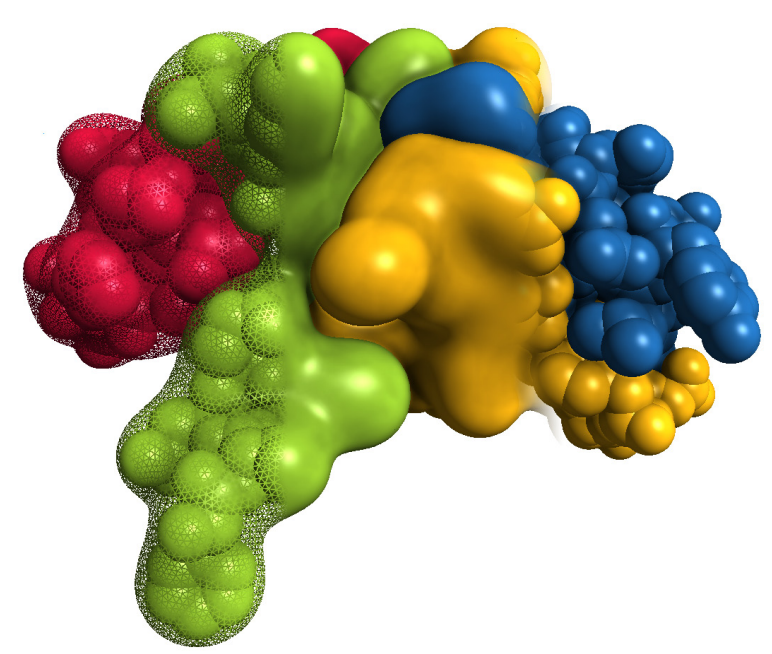

Figure 11: Insulin (PDB-ID: 1RWE) rendered with a surface representation (left and center) and its atomic structure (right).

executed the same test using various other simulation data sets which were different in size but used the same building blocks as the one described in section 6.1. In all our measurements, we observed similar speedups for our optimizations.

\subsection{Mesh Rendering Performance}

We tested the performance of our method using molecular surfaces meshes generated by VMD [HDS96] using the QuickSurf method [KSES12]. In Figure 11, the molecule of insulin (PDB-ID: 1RWE) is depicted with both molecular surface and the underlying structure colored according to protein subunits. On the left, only the edges of the triangle mesh are rendered. The center part shows the molecular surface, whereas the atomic structure is revealed on the far right. We randomly placed the instances of a mesh in a cube (cf. Figure 12). Table 2 shows the measurements for

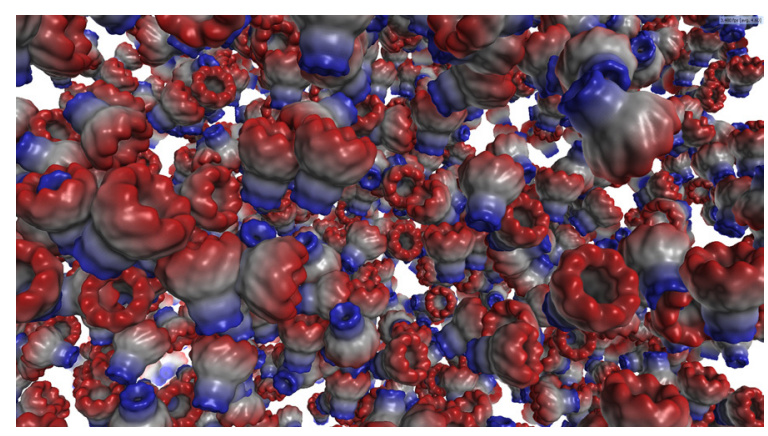

Figure 12: A large number of protein surfaces rendered using our triangle ray marching. a test data set with $220 \mathrm{k}$ triangles. The viewport was set so that all instances were visible. Due to the much higher cost of the triangle intersections compared to the sphere intersections, the performance is significantly lower than that of the sphere rendering. However, our optimized method clearly outperforms OpenGL rendering even when using Vertex Buffer Objects and the OpenGL instancing functionality (up to $100 \%$ speedup). Our test scene is rather sparse since the instances are uniformly distributed. Therefore, the depth culling has only minor influence on the frame rate (especially for lower numbers of instances).

Figure 13 illustrates the difference between atomistic detail and molucular surface rendering applied to the ERK pathway. In the foreground, the general appearance of the protein is depicted more clearly with the surface mesh. Since the normal correction scheme reduces high frequencies for distant objects, the atomistic rendering appears more surface-like and differences in the background are, thus, hardly visible. However, due to the expensive ray-triangle intersection test the surface rendering is slower unless meshes with a low resolution are used.

\section{Future Work}

So far, our simulations do not include information about the rotation of the proteins. However, we apply a biased rotation for rendering which is modeling the rotational diffusion as explained in section 6.1. In the future, we want to add a more refined stochastic model of the rotational diffusion to the underlying simulation which is developed in collaboration with our project partners.

The rendering method we used for our work is restricted to static molecules and triangle meshes. As future work, we want to investigate the feasibility of this algorithm for rendering partly dynamic models which are composed of rigid molecules. One application could be myosin filaments, where only the head domain is moving, or partly rigid MD simulations of viral envelopes, where the capsid proteins are internally rigid but moving.

Table 2: Performance measurements for the triangle rendering. The triangle mesh used for the tests had 220,852 triangles in total. All values are given in frames per second (fps). \#inst denotes the number of instances, \#tria the resulting total number of triangles ( $\mathrm{M}$ denotes millions). The different render modes are as follows: OGL - OpenGL instancing; unopt - our method without optimizations; DC - with depth culling; $\mathrm{VC}$ - with voxel culling.

\begin{tabular}{rrrrrrr}
\hline \#inst & \#tria & OGL & unopt & DC & VC & DC+VC \\
\hline 100 & $22 \mathrm{M}$ & 15.3 & 19.2 & 19.4 & 30.2 & 30.3 \\
500 & $110 \mathrm{M}$ & 3.1 & 3.9 & 4.1 & 5.8 & 6.2 \\
1000 & $220 \mathrm{M}$ & 1.5 & 1.9 & 2.1 & 2.9 & 3.3 \\
2000 & $440 \mathrm{M}$ & 0.8 & 0.9 & 1.1 & 1.2 & 1.4 \\
\hline
\end{tabular}



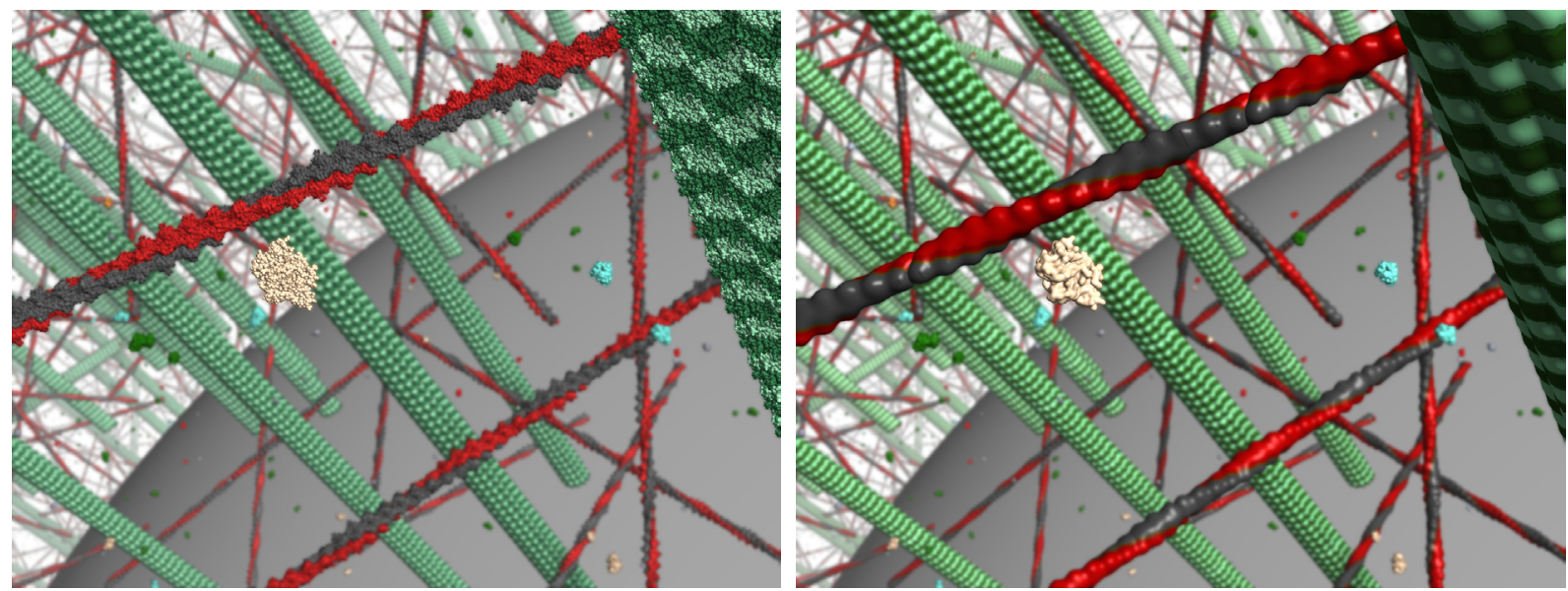

Figure 13: Comparison between atomistic representation (left) and molecular surface meshes (right).

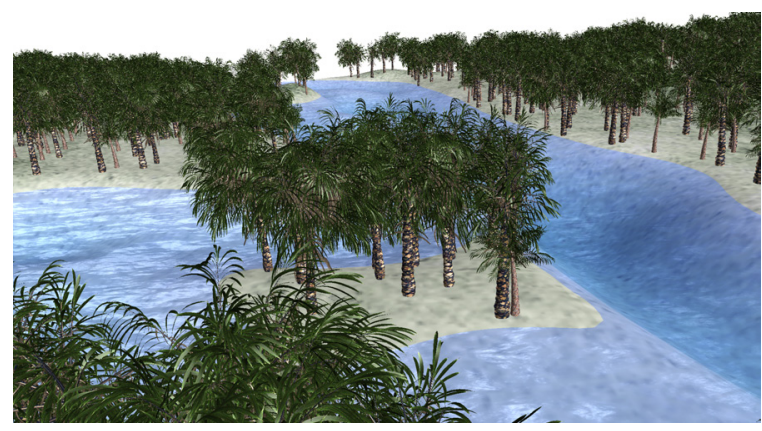

Figure 14: Close-up of the forest test scene including texturing and alpha blending for leaves (65 M triangles, $3.8 \mathrm{fps}$ ).

Another promising direction for future developments would be the usage of tight-fitting bounding geometry. Especially for the filaments and tubules, the currently used bounding box contains many empty cells. Using cylindrical bounding boxes could lead to higher performance while requiring an only slightly more elaborate grid traversal strategy. Other possible bounding geometries would, for example, include spheres, object-aligned bounding boxes or roughly approximated convex hulls.

We believe that our method is also applicable to other fields where many identical instances have to be rendered. One use case could be the rendering of forests. We conducted a first test consisting of 1,000 procedurally generated palm trees (eight tree models, $65 \mathrm{M}$ triangles in total). Our method can render the whole scene (cf. Figure 14) at $3.8 \mathrm{fps}$.

\section{Summary and Conclusion}

We presented a method to visualize mesoscopic whole-cell simulations in atomic detail. This paper is an extended ver- sion of our recent work [FKE12], which is based on the method of Lindow et al. [LBH12]. With our optimized algorithm, it is possible to render the intracellular environment with several billions of atoms at interactive frame rates on current graphics hardware. We modeled the simulated interior of the cell using readily available standard data sets from the Protein Data Bank [BWF*00].

Our application allows the user to apply several methods for image enhancement methods like screen space ambient occlusion [Kaj09] and illustrative, non-photorealistic rendering techniques. These methods can be used to facilitate shape and depth perception. They allow to interactively create visually pleasing, artistic images similar to [Goo], which can be used for instance in publications or as educational resources.

We also extended the GPU ray marching for triangle rendering. This enables rendering not only simple sphere-based molecular models but also complex molecular models like precomputed molecular surfaces (e.g. using the QuickSurf method [KSES12, HDS96]). In our tests, the ray marching method outperforms the standard GPU instancing mechanism for large numbers of instances.

The visualization is integrated into a visual analysis application that is used by our collaborators from the field of systems biology. We presented our results to these project partners and got positive feedback. Amongst other things, they had the impression that this detailed visualization creates a better understanding of cellular dimensions and complexity than simplified representations. The application is available to prospective project partners upon request. In the future, we would like to conduct a formal user study to confirm these anecdotal, preliminary statements. 


\section{Acknowledgments}

This work was partially funded by the German Research Foundation (DFG) within the Cluster of Excellence in Simulation Technology (EXC 310/1) and as part of the Collaborative Research Centre SFB 716 at the University of Stuttgart.

\section{References}

[AM01] AKENINE-MÖLLER T.: Fast 3D triangle-box overlap testing. Journal of Graphics Tools 6, 1 (2001), 29-33. 7

[AW87] Amanatides J., Woo A.: A fast voxel traversal algorithm for ray tracing. In Eurographics (1987), pp. 3-10. 5

[BWF*00] Berman H., Westbrook J., Feng Z., Gilliland G., Bhat T., Weissig H., Shindyalov I., Bourne P.: The protein data bank. Nucleic Acids Research 28 (2000), 235-242. http://www.rcsb.org/. 3,11

[FKE12] Falk M., Krone M., Ertl T.: Atomistic visualization of mesoscopic whole-cell simulations. In Eurographics Workshop on Visual Computing for Biology and Medicine (VCBM) (2012), vol. 2, pp. 123-130. 2, 11

[FKRE09] Falk M., Klann M., Reuss M., ERTl T.: Visualization of signal transduction processes in the crowded environment of the cell. In IEEE Pacific Visualization Symposium (PacificVis) (2009), pp. 169-176. 3

[FOE*11] FalK M., OtT M., ERTl T., KlanN M., Koeppl $\mathrm{H}$.: Parallelized agent-based simulation on CPU and graphics hardware for spatial and stochastic models in biology. In International Conference on Computational Methods in Systems Biology (CMSB 2011) (2011), pp. 73-82. 3

[Goo] Goodsell D.: Molecule of the month. http://www . rcsb.org/pdb/motm. do (Online, Feb. 2013). 7, 11

[GRDE10] Grottel S., Reina G., Dachsbacher C., ERTl T.: Coherent culling and shading for large molecular dynamics visualization. Computer Graphics Forum 29 (2010), 953-962. 2, 5

[GRE09] Grottel S., Reina G., ERTl T.: Optimized data transfer for time-dependent, GPU-based glyphs. In IEEE Pacific Visualization Symposium (Pacific Vis) (2009), pp. 65-72. 2

[GRZ*10] Grottel S., Reina G., Zauner T., Hilfer R., ERTL T.: Particle-based rendering for porous media. In Annиal SIGRAD Conference (2010), pp. 45-51. 3

[Gum03] GumHold S.: Splatting illuminated ellipsoids with depth correction. In International Workshop on Vision, Modeling, and Visualization (2003), pp. 245-252. 2

[HDS96] Humphrey W., Dalke A., Schulten K.: VMD Visual Molecular Dynamics. Journal of Molecular Graphics 14 (1996), 33-38. 7, 10, 11

[HPGK90] Holmes K. C., Popp D., Gebhard W., Kabsch W.: Atomic model of the actin filament. Nature 347, 6288 (1990), 44-49. 4

[HSS*05] Hadwiger M., Sigg C., Scharsach H., BÜHLer K., GROSS M. H.: Real-time ray-casting and advanced shading of discrete isosurfaces. Computer Graphics Forum (2005), 303312. 2

[Kaj09] KaJALIN V.: Screen-space ambient occlusion. In Shader $X^{7}$. Charles River Media, 2009, pp. 413-424. 8, 11

[KSES12] Krone M., Stone J. E., Ertl T., Schulten K.: Fast visualization of gaussian density surfaces for molecular dynamics and particle system trajectories. In EuroVis Short Papers (2012), vol. 1, pp. 67-71. 10, 11
[KW03] KRÜGER J., Westermann R.: Acceleration Techniques for GPU-based Volume Rendering. In IEEE Visualization (2003), pp. 287-292. 2

[LBH12] Lindow N., Baum D., Hege H.-C.: Interactive rendering of materials and biological structures on atomic and nanoscopic scale. Computer Graphics Forum 31, 3 (2012), 13251334. 2, 3, 4, 5, 6, 9, 11

[LBK*07] Lodish H., Berk A., Kaiser C. A., Krieger M., Scott M. P., Bretscher A., Ploegh H., Matsudaira P.: Molecular Cell Biology, sixth ed. W. H. Freeman, 2007. 4

[LD08] LAGAE A., DutRÉ P.: Compact, fast and robust grids for ray tracing. Computer Graphics Forum 27, 4 (2008), 1235-1244. 4

[LHA01] LaW C. C., Henderson A., Ahrens J.: An application architecture for large data visualization: A case study. In IEEE Symposium on Parallel and Large-data Visualization and Graphics (2001), pp. 125-128. 2

[LK10] LAINE S., KARRAS T.: Efficient sparse voxel octrees. In ACM SIGGRAPH 2010 Symposium on Interactive 3D Graphics and Games (2010), pp. 55-63. 3

[LVRH07] Lampe O. D., Viola I., Reuter N., Hauser H.: Two-level approach to efficient visualization of protein dynamics. IEEE Transactions on Visualization and Computer Graphics 13, 6 (2007), 1616-1623. 3

[MT97] Möller T., Trumbore B.: Fast, Minimum Storage Ray-Triangle Intersection. Journal of Graphics Tools 2, 1 (1997), 21-28. 3, 7

[Pal00] PALsson B.: The challenges of in silico biology. Nature Biotechnolgy 18, 11 (2000), 1147-1150. 3

[PC81] Potmesil M., ChaKRaVARTy I.: A lens and aperture camera model for synthetic image generation. Computer Graphics (Proceedings of SIGGRAPH 1981) 15, 3 (1981), 297-305. 8

[PSQH06] Pogson M., SMallwood R., Qwarnstrom E., Holcombe M.: Formal agent-based modelling of intracellular chemical interactions. Biosystems 85, 1 (2006), 37-45. 3

[SMLL*10] SHAW D. E., MARAGAKIS P., LiNDORFF-LARSEN K., Piana S., Dror R. O., Eastwood M. P., Bank J. A., Jumper J. M., SAlmon J. K., Shan Y., Wriggers W.: Atomic-level characterization of the structural dynamics of proteins. Science 330, 6002 (2010), 341-346. 3

[SP12] ShUMSKIY V., PARSHIN A.: Gpu ray tracing - comparative study of ray-triangle intersection algorithms. In International Conference on Computer Graphics and Vision (2012). 3

[ST90] SAITO T., TAKAhASHI T.: Comprehensible rendering of 3-d shapes. Computer Graphics (Proceedings of SIGGRAPH) 24, 4 (1990), 197-206. 7

[TCM06] Tarini M., Cignoni P., Montani C.: Ambient occlusion and edge cueing for enhancing real time molecular visualization. IEEE Transactions on Visualization and Computer Graphics 12, 5 (2006), 1237-1244. 7

[Wal04] WALD I.: Realtime Ray Tracing and Interactive Global Illumination. PhD thesis, Computer Graphics Group, Saarland University, 2004. 3

[WFP*01] WAND M., Fischer M., Peter I., Meyer Auf Der HEIDE F., STRASSER W.: The randomized z-buffer algorithm: interactive rendering of highly complex scenes. In ACM SIGGRAPH Computer Graphics and Interactive Techniques (2001), pp. 361-370. 3 